\title{
Análisis de la forma de onda de señales de Emisión Acústica producida en ensayos de interfaces ceramo-metálicas
}

\section{Analysis of Acoustic Emission waveform signals produced in test on ceramo- metallics interfaces}

\author{
Valeria Inés Ortega Paredes ${ }^{1,2}$, Martín Pedro Gómez ${ }^{1,2,3}$, \\ Leandro Dócimo ${ }^{4}$, Nicolás Nieva ${ }^{5}$, María Isabel Lopez Pumarega ${ }^{1}$
}

\author{
${ }^{1}$ Dpto. ICES, Comisión Nacional de Energía Atómica (CNEA), Buenos Aires, Buenos Aires, Argentina \\ e-mail: vortega@cnea.gov.ar \\ ${ }^{2}$ IT Sabato, UNSAM-CNEA, San Martín, Buenos Aires, Argentina \\ ${ }^{3}$ CENES, FRD, UTN, Campana, Buenos Aires, Argentina \\ e-mail: mpgomez@cnea.gov.ar \\ ${ }^{4}$ Cátedra de Materiales Dentales, Hospital Odontológico Universitario, Facultad de Odontología, Universidad de Buenos \\ Aires, Buenos Aires, Buenos Aires, Argentina. \\ ${ }^{5}$ Universidad Nacional de Tucumán, Facultad de Ciencias Exactas y Tecnología, Departamento de Física, Laboratorio de \\ Física Del Sólido, Tucumán, Argentina.
}

\section{RESUMEN}

El estudio de las interfaces ceramo-metálicas es de particular importancia en odontología dada la demanda actual de las prótesis dentales, cada vez más parecidas a las piezas dentales naturales y de vida útil extensa. El objetivo del presente trabajo es profundizar el estudio del comportamiento de éstas interfaces para sistemas de restauración dental y así caracterizar la adherencia del cerámico (porcelana feldespática) al metal (aleaciones de base no noble, como cromo (Cr) comúmente utilizadas en odontologia) por medio de la técnica de ensayos no destructivos denominada Emisión Acústica (EA). Para ello, se fabricaron probetas bajo Norma, formadas por un sustrato metálico, de aleación de $\mathrm{NiCr}$ y un depósito de porcelana feldespática. Parte de los sustratos de dicho grupo de probetas fueron sometidos a distintas combinaciones de tratamientos superficiales tales como arenado y tratamientos térmicos, previamente a realizar el depósito cerámico. Estos tratamientos forman parte del proceso de fabricación de las prótesis y ambos buscan optimizar la unión de la porcelana a la aleación. Cada probeta fue ensayada bajo Norma a la flexión en un dispositivo de tres puntas hasta producir el desprendimiento del cerámico, y se examinó dicho fenómeno a tiempo real a través de la EA. Durante estos ensayos se obtuvieron las formas de onda de la EA y sus parámetros. La señal de la carga aplicada permitió detectar y estudiar los parámetros más representativos para cada probeta. Se realizó el análisis de las formas de onda de las señales de EA, estudiando los eventos producidos en momentos relevantes del proceso de desprendimiento de la interfaz. En este caso se usaron características de la forma de ondas de las señales de EA, en particular un parámetro proporcional a la energía de las ondas P con el fin de clasificarlas para distintas condiciones de adherencia.

Palabras clave: interfaces, adherencia, Emisión Acústica, forma de onda, desprendimiento.

\section{ABSTRACT}

The study of ceramic-metal interface is of special importance in odontology area by the current demand about dental prostheses, so it is very important to be more and more similar to natural teeth and with a long service life. With the aim of assessing in detail the behavior of ceramic-metal interface used in dental restorations systems, in this work the adherence between ceramic (feldspathic porcelain) and metal (chrome-based alloys frequently used in odontology) is characterized through a non-destructive tests method called Acoustic Emission (AE). For this, samples were made under a Standard, with a Ni-Cr alloy substrate and feldspathic porcelain layer. The substrates were subjected to different surface treatments, such as sand blasting and thermal treatments, before applying the ceramic layer. These treatments take part of the manufacturing pro- 
cess and allow improving the bonding between porcelain and alloy. Each sample was tested under a specific Standard with bending on a three-point device until the ceramic became detached. The detachment was studied with $\mathrm{AE}$ in real time. The $\mathrm{AE}$ waveforms and the parameters load signal were obtained during the tests. The analysis of the AE signal waveforms was made by studying the events resulting from the most important moments of the interface-debonding process. In this case, waveform features of the AE signals were used, in particular a parameter proportional to the energy of the $\mathrm{P}$ waves in order to classify them as different conditions of adhesion.

Keywords: interfaces, adherence, Acoustic Emission, waveform, debonding.

\section{INTRODUCCIÓN}

Según estudios previos sobre sistemas de restauración dental, especialmente aquellos de porcelana fundida sobre metal (PFM), los cuales involucran interfaces ceramo-metálicas, se sabe que la adherencia es optimizada por medio de la aplicación de tratamientos térmicos (TT) y superficiales sobre el substrato metálico, entre otros los citados en [1, 2, 3]. El éxito de la unión entre el cerámico y el metal depende de: 1) la capacidad de la aleación de formar óxidos superficiales solubles en el vidrio de la porcelana, (por medio de un TT sobre el sustrato) 2) la mayor similitud entre el coeficiente de variación dimensional térmica de ambos materiales, 3) la temperatura de fusión del metal, ésta debe ser muy elevada respecto a la correspondiente de la porcelana pues así se evita el efecto de creep que sufre el metal al fundir la porcelana por medio de tratamientos térmicos llamados cocciones sobre la aleación y 4) el módulo elástico del metal, que debe ser suficiente para brindar la rigidez necesaria al conjunto, según la porcelana a utilizar. La adherencia de estos sistemas es estudiada según la Norma IRAM ISO 9693, específica para Sistemas Metal-Cerámicos de Restauración Dental [4, 5, $6,7,8,9]$.

En este trabajo se estudia dicha interfaz por medio de la técnica de Emisión Acústica (EA), a través de la forma de onda de las señales de EA. El método es aplicado a ensayos según la Norma mencionada anteriormente. Para esto utilizamos 8 probetas, seleccionadas al azar de un grupo de 16 probetas. Estas consisten en un depósito cerámico sobre un sustrato metálico al cual se le realizó, previo al depósito del cerámico, en unos casos un tratamiento térmico, en otros un arenado y en otros casos ambos.

Las cerámicas dentales utilizadas actualmente para este tipo de restauraciones requieren de una fase vítrea, el feldespato, que aporta la translucidez y de una fase cristalina constituida por cuarzo, que eleva la temperatura de fusión y la resistencia. Además, contienen caolín, que facilita la manipulación de la porcelana cuando aún no ha sido cocida, fundentes que bajan la temperatura de sinterizado y pigmentos para lograr las diferentes tonalidades de las piezas dentales según el paciente. Estas cerámicas o porcelanas feldespáticas, poseen buenas propiedades ópticas, lo que beneficia el aspecto estético de las piezas pero son frágiles y por lo tanto, para ser aplicadas en prótesis dentales deben ser depositadas sobre un apoyo o una estructura de soporte, es por eso que se las usa para recubrimientos de estructuras metálicas o cerámicas. En éste trabajo, se utilizaron como estructuras metálicas, sustratos de CoCr.

Respecto a la Técnica de EA, ésta consiste en someter el material de interés, en nuestro caso el conjunto ceramo-metálico, a una solicitación (tensión, temperatura, etc.) provocando un cambio en el campo de tensiones del material, tal que la onda mecánica resultante se propague hasta alcanzar su superficie y allí sea detectado por los sensores del sistema de EA $[10,11]$.

Por medio del sistema de EA obtuvimos los parámetros característicos de la EA de estos ensayos y la forma de onda de cada evento detectado. La forma de onda es estudiada en este trabajo con el objetivo de observar detalles que permitan ver cómo afectó cada tratamiento sobre el sustrato de cada probeta a la estabilidad de la interfaz.

\section{MATERIALES Y MÉTODOS}

\subsection{Obtención de las probetas}

El sustrato metálico se fabricó a partir de aleación $\mathrm{Cr}-\mathrm{Ni}$, comúnmente usada para restauración de cerámicas dentales. La composición de la aleación fue la siguiente: Ni 77,95\%, Be 1,95\%, Cr 12,60\%, Mo 5\%, Al 2,9\%, Co $0,45 \%$.

El cerámico empleado fue una porcelana feldespática cuyo coeficiente de expansión térmico fue el apto para ser utilizado sobre aleaciones $\mathrm{Cr}-\mathrm{Ni}$, ya que éste es un factor determinante de la adherencia [1, 2, 3]. 
Se conformaron 4 grupos según el criterio basado en la combinación de tratamientos que sufrió el sustrato metálico (aleación $\mathrm{Cr}-\mathrm{Ni}$ ) previo al depósito del cerámico, es decir si fue o no arenado y si sufrió o no TT.

Las probetas fueron seleccionadas en principio de un grupo de 16 probetas, denominadas $\mathrm{P} 1, \ldots, \mathrm{P} 16$, del cual se formaron 4 grupos de 4 probetas cada uno. Una probeta de cada grupo fue apartada como "testigo”. Así, se denominó Grupo SASTT (P1,.., P4) a las probetas con sustratos sin arenado y sin TT, del cual se eligieron al azar las probetas P2 y P3. El Grupo SACTT (P5,.., P8) corresponde a sustratos sin arenado con TT, del que se tomaron las probetas P6 y P7. El Grupo CASTT (P9,.., P12) con sustratos arenados sin TT, del cual se eligieron a P9 y P10. El grupo CACTT $(\mathrm{P} 13, \ldots, \mathrm{P} 16)$ con sustratos arenados y con TT del cual se eligieron las probetas P13 y P14.

Las dimensiones de las probetas, cuya forma es rectangular, son las que se indican en la Figura 1 siguiendo indicaciones de la normativa mencionada [2, 3, 4].

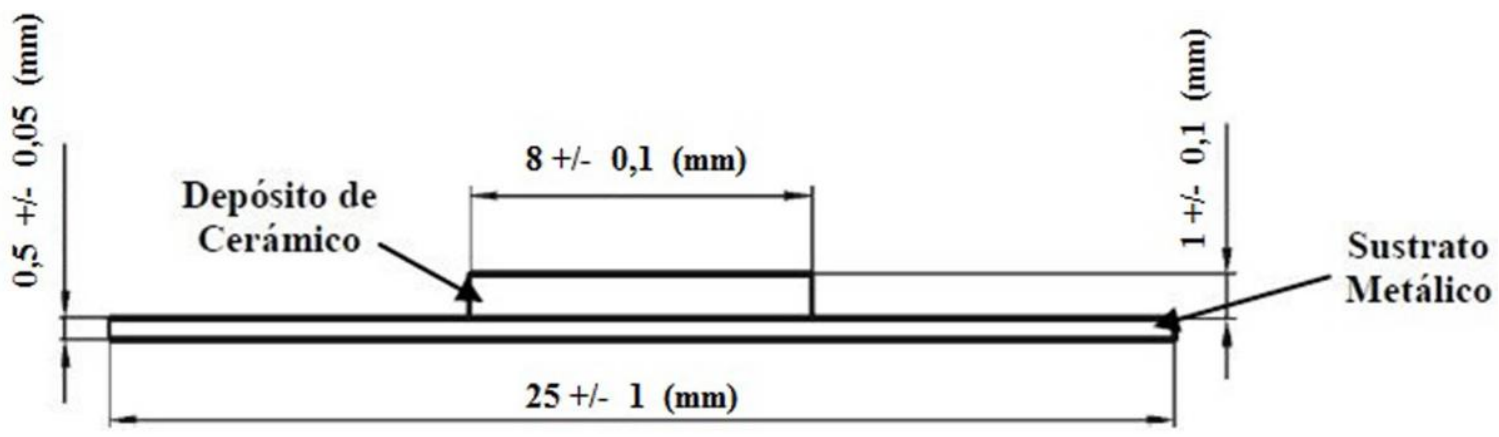

Figura 1: Dimensiones de la probeta según la Norma IRAM ISO 9693.

\subsection{Técnica de Emisión Acústica}

El fenómeno de EA se puede definir según la Norma EN 1330-9:2000 [12], como el término utilizado para las ondas elásticas transitorias generadas por la liberación de energía en un material o producida por un proceso. Las Normas ASTM 1316-99 [13] e IRAM 779 [14] la definen como la clase de fenómeno por el cual las ondas elásticas se generan por la liberación rápida de energía desde fuentes localizadas dentro del material o las ondas transitorias así producidas.

La EA se caracteriza por un rango de frecuencias de $10 \mathrm{KHz}$ a $1 \mathrm{MHz}$. Es un método de ensayos no destructivos (END) considerado comúnmente "pasivo" dado que le material al ser excitado por medio de una solicitación produce EA a diferencia del ultrasonido, por medio del cual el mismo material recibe y emite US.

La técnica consiste en la aplicación de una solicitación sobre el material bajo estudio, GOMEZ, M. P. [11]. Un sistema básico de EA, en general se puede describir como se muestra en la figura 2. En este caso, se aplica una carga la cual provoca un cambio en el campo de tensiones de la muestra generando una microfisura asociada al despegado del cerámico y la posterior propagación de una onda mecánica. Cuando la onda mecánica llega a la superficie del material impacta a modo de golpe (hit) sobre el sensor de EA, generalmente un piezoeléctrico (PZT), transformado la energía mecánica en eléctrica para poder procesarla como una señal eléctrica.

Este método permite estudiar la señal de EA obtenida, desde dos aspectos. Uno corresponde a sus parámetros característicos y otro a la forma de onda de los eventos de interés. 


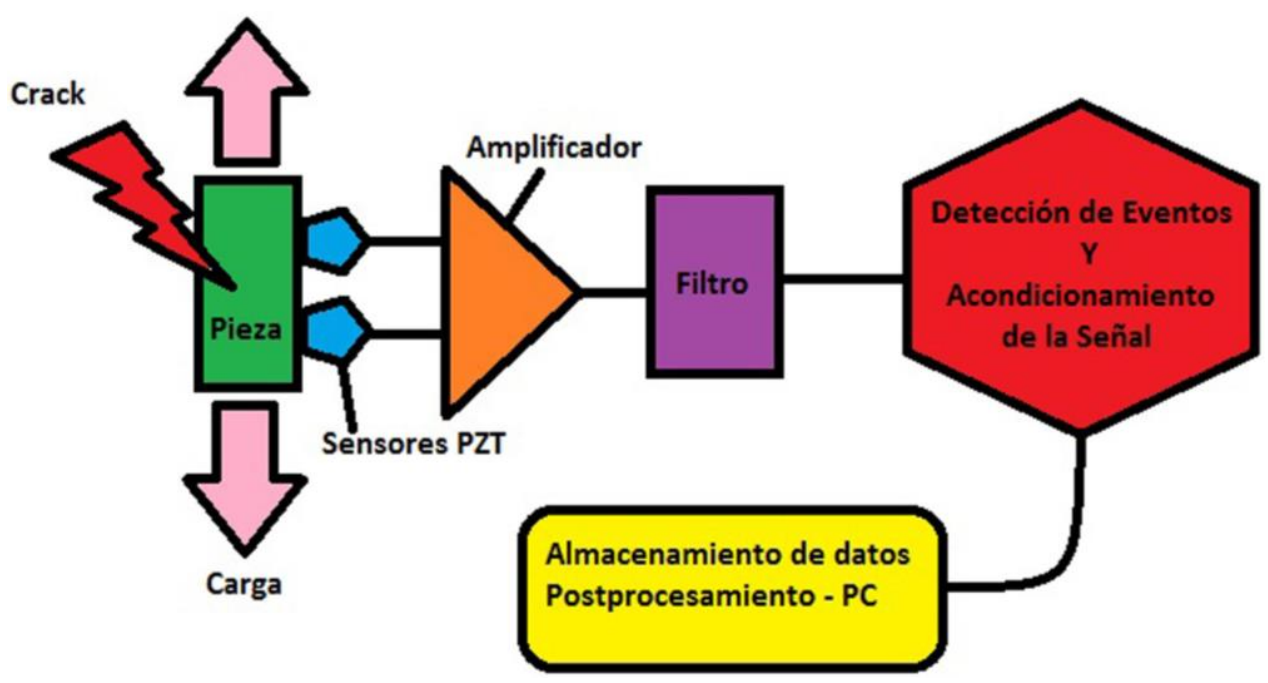

Figura 2: Diagrama de un Sistema de EA.

\subsection{Realización de los ensayos y medición de la EA}

Los ensayos se realizaron a través de un sistema conjunto conformado por una parte mecánica de carga y por el sistema de EA. El sistema mecánico consistió en una punta que descendía a una velocidad constante de 1,5 $\mathrm{mm} / \mathrm{min}$ hasta alcanzar el sustrato metálico de la probeta. Los extremos de la probeta se colocaron sobre 2 apoyos correspondientes a un soporte cuya base se ubicó directamente sobre la celda de carga modelo CDL-5 con rango de trabajo es de 0 a $6 \mathrm{kgf}[2,3,10]$. Esta celda permitió observar la fuerza a la cual se producía el desprendimiento del cerámico respecto del metal y al mismo tiempo detectar el evento de EA característico del despegue. La salida de la celda de carga se conectó como parámetro externo al sistema de medición como se muestra en la figura 3. El equipo de 2 canales de AE, está constituido por una placa marca PAC modelo PCI-2, con un conversor A/D de 18 bits y una tasa máxima de muestreo de $40 \mathrm{Ms} / \mathrm{s}$. Ambos canales se conectaron a pre-amplificadores de $40 \mathrm{~dB}$ ganancia. Para detectar las señales se utilizaron dos sensores, uno de banda ancha $(1 \mathrm{kHz}$ a $1 \mathrm{MHz})$ y otro resonante R15D $(150 \mathrm{kHz})$. Los sensores se colocaron en las paredes externas del soporte de la probeta. A la derecha se colocó el de banda ancha, conectado al canal 1 de la placa y a la izquierda el resonante, conectado al canal 2, ambos usando un acoplante acústico adecuado. El sensor cuya señal es de interés para el presente trabajo es el de banda ancha ya que este permite un análisis óptimo de la forma de onda de las señales de EA.

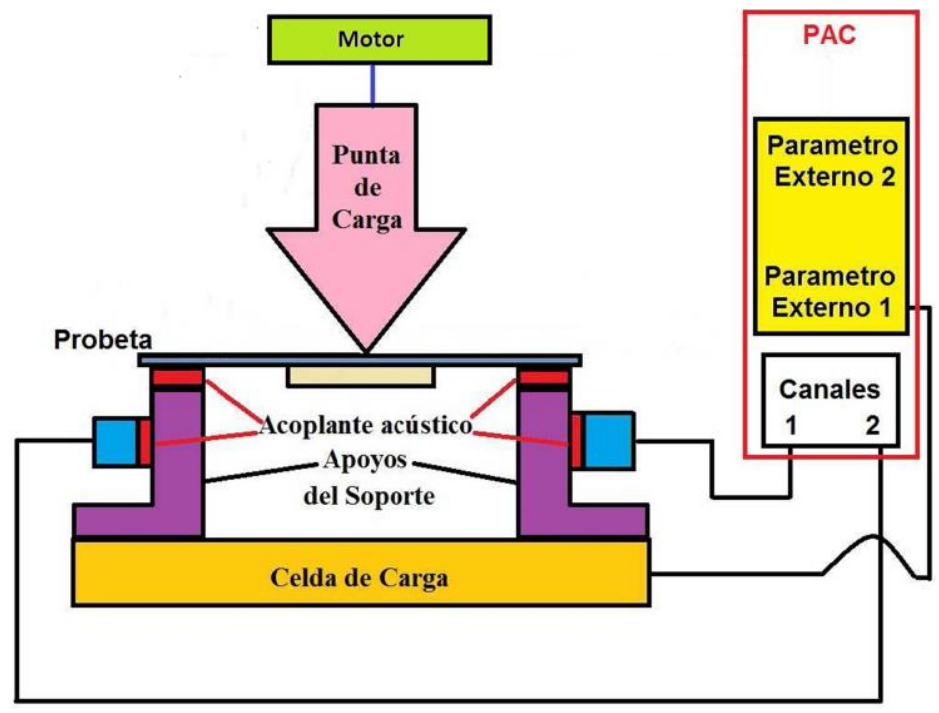

Figura 3: Diagrama del Sistema de Medición.

\subsection{Estudio de la forma de onda de la señal de EA. Ondas P y S.}

La señal de EA obtenida a través del sensor es originalmente una onda elástica que se propaga en un medio 
sólido. A partir de aplicar los fundamentos de la mecánica del continuo a un sólido lineal, elástico, isótropo e infinito, considerando teoremas de conservación, suposición de elasticidad de Green y la relación de Cauchy para pequeñas deformaciones, si se aplica un campo de tensiones a un cubo infinitesimal se obtienen dos ecuaciones de propagación de ondas elásticas. Una es escalar y representa a las ondas P y otra es vectorial y describe las ondas S. De esa solución salen las ecuaciones que representan las velocidades de ambas (Ecuaciones- 1 y 2 ).

$$
\begin{aligned}
& V_{L}=\sqrt{\frac{E(1-\sigma)}{\rho(1-\sigma)(1-2 \sigma)}} \\
& V_{T}=\sqrt{\frac{E}{2 \rho(1+\sigma)}}
\end{aligned}
$$

Donde:

$\mathrm{V}_{\mathrm{L}}$ : es velocidad de ondas longitudinales o $\mathrm{P}$.

$E$ : es el módulo de Young, una constante que depende del material.

$\sigma$ : es el cociente de Poisson, un parámetro adimensional comprendido entre 0 y 0.5 , que depende del material.

p: es la densidad del sólido.

$V_{T}$ : velocidad de ondas transversales o $\mathrm{S}$

Si calculamos la relación entre ambas, $V L / V T$, en general para metales como los utilizados en este trabajo ésta resulta próxima a 2. Esto significa que las ondas $\mathrm{P}$ son más rápidas que las $\mathrm{S}$ y por lo tanto, son las que llegan primero a la superficie. Entonces cuando se produce una fuente que genera ondas de EA, las ondas P serán las primeras en llegar al sensor. Hay otros tipos de ondas (o fases) que se producen cuando existe una interface en un medio no infinito. Por ejemplo, en un medio semi-infinito aparecen las ondas de superficie o Rayleigh, y en un medio tipo guía de onda (placas o tubos) aparecen las ondas de Lamb. Las ondas $\mathrm{P}$ por ser las más veloces permanecen inalteradas pero las siguientes se verán afectadas por las fases más lentas y las reflexiones, que pueden generar información confusa. Por este motivo, en este caso se eligen las ondas P para el análisis realizado de la señal de EA del evento detectado durante el inicio del desprendimiento del depósito cerámico, GOMEZ, M. P. [11].

\section{RESULTADOS Y DISCUSIÓN}

En trabajos previos donde se estudiaron diferentes grupos de probetas, enfocando el análisis a la parte paramétrica de la EA se encontró que el parámetro de Amplitud era el que se destacaba en el momento del despegue del cerámico [2,3]. Se observó que en el momento donde se iniciaba el desprendimiento, al caer la carga, se generaba un evento de amplitud próxima a $93 \mathrm{~dB}$ en todos los casos. En la figura 4 se muestra un ejemplo por medio de una gráfica dónde se representa la Amplitud en dB (rosa) y la Carga en Kgf (azul), ambas como funciones del tiempo de ocurrencia expresado en segundos.

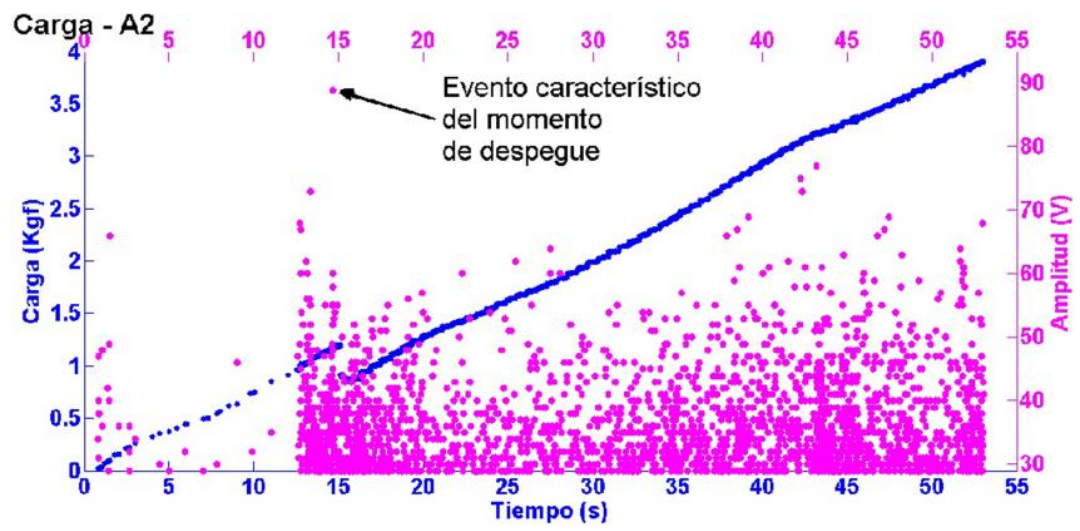

Figura 4: Evento característico del inicio del desprendimiento del cerámico en la gráfica de Amplitud y Carga versus tiempo.

Se realizó la identificación de dicho evento y se lo comparó con eventos anteriores y posteriores se- 
leccionados para cada probeta luego de la aplicación de filtros empleados según un rango de amplitudes, tiempos mínimos de llegadas al sensor de banda ancha, valores de carga idénticos, y el resto de parámetros tales cómo el tiempo de subida, el número de cuentas, la energía, la duración, el RMS, la energía absoluta [2,3]. Luego, se observó detalladamente la forma de onda del evento característico del despegue del cerámico y se graficó la amplitud de la onda P. En la figura 5 se muestra la forma de onda de la EA correspondiente al evento característico para cada probeta. Varias gráficas de esta figura, muestran amplitudes máximas que oscilan entre $4 \mathrm{mV}$ y $6 \mathrm{mV}$. Durante la observación de las formas de ondas del evento de interés de cada probeta, se pudo ver que la probeta P10 del grupo CACTT y la probeta P14 del grupo CASTT presentaron formas de onda muy diferentes respecto al resto, ver figura 5. Esto pudo deberse a un error del disparo del sistema de EA al saturar la señal y por lo tanto hubo que descartar esas señales, dejando así cada uno de los dos grupos mencionados sólo con una probeta confiable para el análisis.

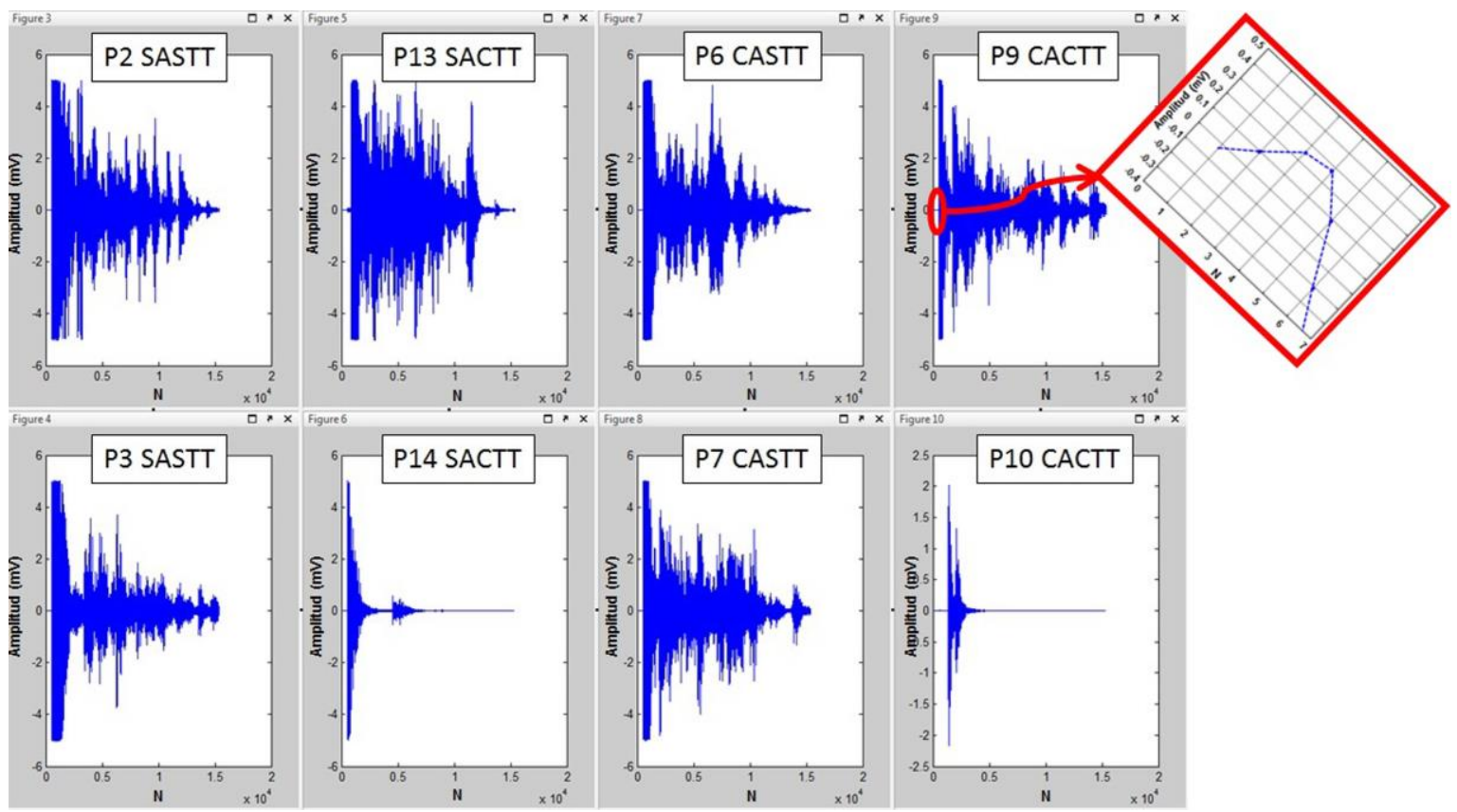

Figura 5: Forma de onda de la EA del evento característico $(93 \mathrm{~dB})$ para cada probeta. Se graficó la Amplitud en función de la cantidad de puntos (N). En la gráfica de P9 se observa una ampliación del inicio de la señal de EA para distinguir la onda P.

\subsection{Reconstrucción de la onda $\mathbf{P}$}

A partir de la identificación de la onda $\mathrm{P}$ se midió un valor proporcional a la energía MARSE (área medida bajo la curva rectificada de la señal) a partir del cual se caracterizó la magnitud de las ondas producidas durante el inicio del despegue del cerámico, el cual ocurre de manera abrupta. Se ha visto en trabajos anteriores que en ese momento se genera un evento cuyos hits son de gran amplitud. En algunos ensayos hechos en este trabajo, las señales del despegue eran de mayor amplitud que el rango de medición del equipo por lo que se produjo la saturación de la parte más energética de la señal, correspondiente a las ondas de superficie o Rayleigh (R), pero no ocurrió eso para las ondas $\mathrm{P}$, de menor amplitud. Luego de la P llegan las fases más lentas, sucesivas reflexiones y oscilaciones del sensor.

Para poder identificar las ondas $\mathrm{P}$ se utilizó un criterio basado en el arribo de esas ondas y de las posteriores ondas de corte (S) y R. Para cada hit muestreado, correspondiente al momento del despegue, se asumió la velocidad de propagación de las ondas $\mathrm{P}$ en un valor estimado de $5000 \mathrm{~m} / \mathrm{s}$, y de las ondas $\mathrm{S}$ de la mitad de ésta. Entonces se identificaron en las capturas de estas señales ambas fases $\mathrm{P}$ y $\mathrm{S}$ y se seleccionó el primer hemiciclo de éstas para "medir la energía" para todos los casos. La distancia aproximada que viaja la onda mecánica desde la fuente de EA, que se asume generada en el despegue en uno de los dos lados del depósito cerámico, hasta llegar al sensor de Banda Ancha, es de 0,04 m. Con estos datos se estimó el tiempo que tardaría la onda $\mathrm{P}$ en llegar al sensor, y resultó de 8 microsegundos respectivamente y ese mismo tiempo es el lapso hasta la llegada de la onda S. De esa manera se estimó con precisión la onda P "pura”.

El valor de la frecuencia de muestreo con la cual se realizaron las mediciones fue de $2 \mathrm{Ms} / \mathrm{s}$, esto quie- 
re decir que entre muestra y muestra hay 0,5 microsegundos. Con los datos mencionados se logró identificar la onda $\mathrm{P}$ de forma aproximada. Luego se calculó un valor en voltaje $\left(A_{p}\right)$ proporcional a la MARSE $\left(E_{p}\right)$ de la onda P. Esto se hizo sumando el voltaje de cada muestra $\left(A_{i}\right)$ a lo largo de la onda P (ver Ec. 3 y 4 ).

$$
\begin{gathered}
E_{P}=\sum_{i} A_{i} \cdot \Delta t_{i}=\Delta t_{i} \sum_{i} A_{i} \\
A_{P}=\frac{\Delta t_{i} \sum_{i} A_{i}}{\Delta t_{i}}=\sum_{i} A_{i}
\end{gathered}
$$

Donde $i$ representa cada muestra tomada de la curva, $A_{i}$ las correspondientes amplitudes de la señal (en $\mathrm{mV}$ ) y $\Delta t_{i}=0,5$ microsegundos, el intervalo de tiempo entre muestras consecutivas.

A cada muestra que conforma la curva de la onda $\mathrm{P}$ le corresponde un valor de tensión en $\mathrm{mV}$ dado según la Ec. 4. Para cada grupo de probetas se obtuvieron las representaciones de la onda P individualizadas, como se muestra en la Figura 6.

\section{SASTT}

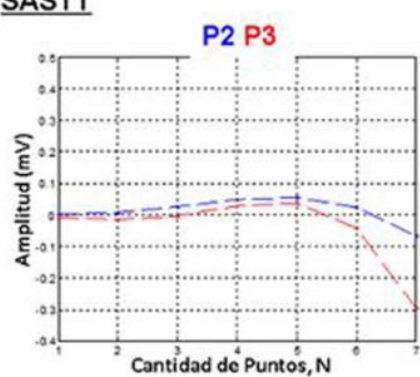

\section{CACTT}

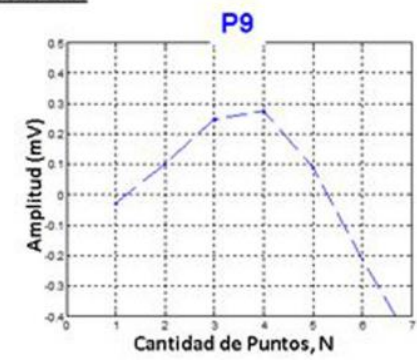

SACTT

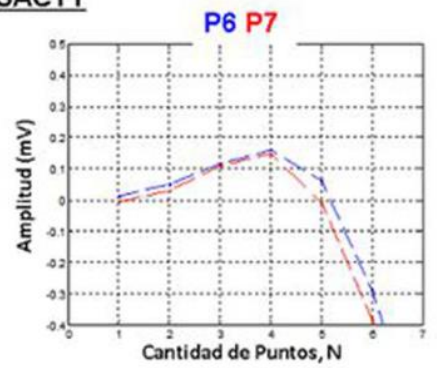

CASTT

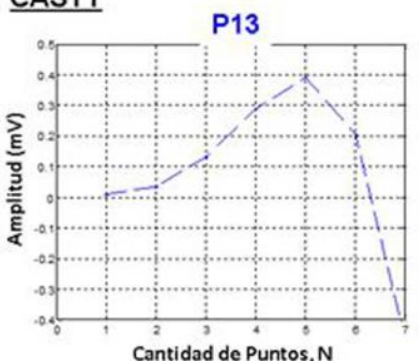

Figura 6: Onda $\mathrm{P}$ correspondiente a la señal de EA del evento característico para cada grupo de probetas.

Luego se calculó el promedio de dicha amplitud para los casos de las dos probetas agrupadas en SASTT y SACTT. La Tabla 1 muestra los resultados obtenidos para cada grupo.

Tabla 1: Amplitudes AP de cada onda P para cada probeta y Amplitud AP PROM, para cada grupo.

\begin{tabular}{c|l|l|l|l|l}
\hline \multicolumn{1}{c|}{ GRUPO } & PROBETA & A P ( MV) & A P PROM ( MV ) & CARGA DESP.( KGF) & CARGA DESP. PROM. (KGF) \\
\hline \multirow{4}{*}{ SASTT } & P2 & 0,16 & & 1,24 & \\
\cline { 2 - 3 } & P3 & 0,06 & 0,11 & 1,04 & 1,14 \\
\hline \multirow{3}{*}{ SACTT } & P6 & 0,40 & & 1,68 & \\
\cline { 2 - 3 } CACTT & P7 & 0,28 & 0,34 & 1,35 & 1,51 \\
\hline CASTT & P13 & 0,72 & 0,72 & 1,36 & 1,36 \\
\hline
\end{tabular}




\subsection{Comparacón entre emisión acústica y carga según el grupo de probetas}

De los resultados obtenidos del promedio de la sumatoria de las amplitudes calculada para cada onda P de cada probeta, y de los obtenidos de la celda de carga en el momento del inicio del despegue del cerámico, carga de despegue para cada probeta y la carga promedio de inicio del despegue para cada grupo, se graficó la curva de la figura 7.

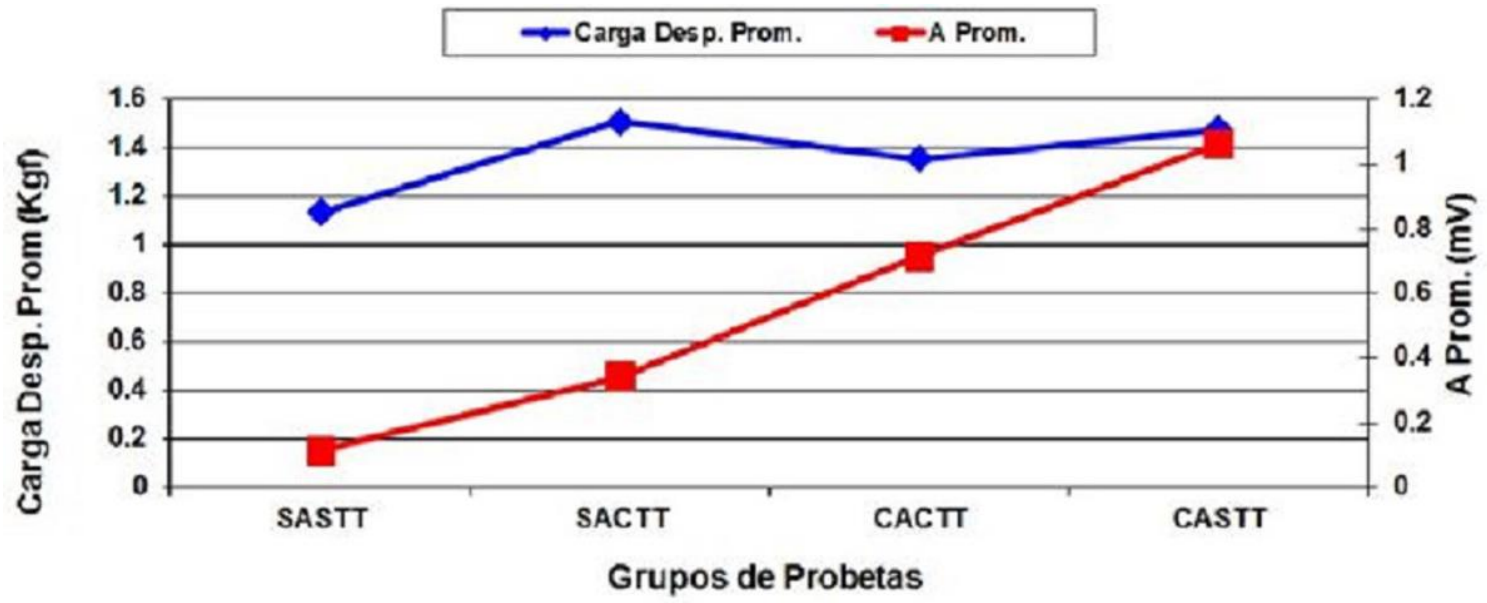

Figura 7: Amplitud total de la onda P, calculada para cada probeta y promediada, para aquellos grupos con más de una probeta y Carga de despegue promedio, en función de cada grupo de probetas.

Se puede inferir, observando la figura 6, que la amplitud promedio de la onda $\mathrm{P}$ para cada probeta es mayor para la probetas de sustratos con arenados que para aquellas que sólo sufrieron tratamiento térmico y de la figura 7, que el arenado es el tratamiento determinante para una mejor adhesión del cerámico al metal, como se ve en la curva de amplitudes; en cuanto al tratamiento térmico se puede decir que mejora la adhesión pero no de forma tan significativa como lo hace el arenado. Lo mismo se puede inferir del análisis de las cargas de despegue, es decir con los datos obtenidos de la celda de carga. Ambas curvas presentan una tendencia parecida. Por medio del estudio de la forma de onda correspondiente al momento de despegue se puede analizar la adherencia del conjunto ceramo-metálico de forma más efectiva que con el estudio de la carga o fuerza a la cual se inicia el desprendimiento del cerámico, debido a la mayor sensibilidad de la técnica de EA.

Respecto a los tratamientos aplicados sobre el sustrato metálico, se podría decir que el tratamiento térmico no fue tan efectivo como el arenado. Esto podría deberse a que el primero favorece la formación de óxidos superficiales que son solubles en la fase vítrea del cerámico, esto en principio, optimiza la unión en cuestión. Esta capa respondería a una cierta estabilidad que depende de la cantidad de óxido superficial producido por la aleación no noble durante el tratamiento térmico. Es probable que el tratamiento realizado en este caso, fuera excesivo y por ello la cantidad de óxido resultante superó el límite necesario para una unión óptima, lo que redujo el grado de adherencia en la interfaz. Es por eso que, al observar ambas curvas (roja y azul) en la figura 7, aquellas probetas en las que se combinaron tanto arenado como tratamiento térmico resultaran de interfaces menos resistentes a la flexión que aquellas donde sólo se realizó arenado sobre el sustrato, pues se sabe que éste último aumenta la superficie de contacto entre el cerámico y el metal y aparentemente, esté fue de mayor aporte a la adhesión.

\section{CONCLUSIONES}

En el presente trabajo, por medio del uso del método de EA se logró estudiar con eficacia la adherencia de sistemas ceramo-metálicos, implementando el análisis de la forma de onda de la señal de EA obtenida durante el inicio del desprendimiento del cerámico. En especial, se estudió la onda $\mathrm{P}$ de dicha señal ya que ésta se encuentra libre de interferencias de otras ondas que se propagan en el material por el hecho de ser la primera en llegar a la base del sensor PZT. De esta onda se estudió el semiciclo positivo por medio de la sumatoria de las amplitudes, punto a punto, obteniendo así un valor de amplitud representativo para cada onda P. Este valor se propone como una posible variable de caracterización de la interfaz en estudio, pues permit iría indicar el grado de adherencia en este tipo de probetas.

Por medio de este procedimiento se pudo observar que, para la mejora de la unión de la interfaz, el tratamiento de arenado sobre el sustrato metálico es más efectivo que el tratamiento térmico, en principio, podría deberse a un exceso en la producción de óxidos superficiales durante el último, situación que no favo- 
rece la adhesión. Al comparar lo obtenido por la Técnica de EA con lo obtenido de la celda de carga, método con el que comúnmente se realizan estos ensayos, se podría decir que la EA es más precisa dada la sensibilidad de la técnica, pues la Norma 9693 para Sistemas de Restauración Dental, realiza estos ensayos detectando el desprendimiento del metal a ojo desnudo y a oído, herramientas poco precisas y con un gran error durante la detección. Implementar la Técnica de EA en dicha Norma sería de contribución importante al estudio y caracterización de estas interfaces, con el objetivo de optimizar la producción de prótesis dentales por medio de la técnica de porcelana fundida sobre metal.

Se propone repetir este análisis en grupos más numerosos de probetas para corroborar los resultados obtenidos en este trabajo.

\section{BIBLIOGRAFÍA}

[1] CARRIZO, R. N., "Evaluación de la Resistencia al despegado de Interfaces Metal/Cerámico en Materiales de Uso Odontológico", Tesis de Grado, Universidad Nacional de Tucumán, Fac. de Ciencias Exactas y Tecnología, Tucumán, Argentina, 2011.

[2] ORTEGA PAREDES, V. I. , KAPLAN, A. , GÓMEZ, M. P., et al., "Characterization of Metal/Ceramic Interfaces in Dental Materials by Acoustic Emission”, Procedia Materials Science, v. 8, pp. 683-692, 2015.

[3] ORTEGA PAREDES, V.I., LOPEZ PUMAREGA, M.I., NIEVA, N., et al., "Adhesion study in MetalCeramic Systems of Dental Restoration by Acoustic Emission”, In Procedia Materials Science, v. 9, pp. 477483, 2015 .

[4] PROYECTO 1 DE NORMA ARGENTINA. Norma IRAM ISO 9693, “Sistemas Ceramo-Metálicos para Restauración Dental", Primera edición, 2008.

[5] XIANG, N., XIN, X. Z., CHEN, J., WEI, B., "Metal-ceramic bond strength of Co-Cr alloy fabricated by selective laser melting”, Journal of Dentistry, v. 40, pp. 453-457, 2012.

[6] LEE, S. B., LEE, J. H., KIM, W. C., et al., "Effect of different oxidation treatments on the bonding strength of new dental alloys", Thin Solid Films, v. 517, pp. 5370-5374, 2009.

[7] HENRIQUES, B., FARIA, S., SOARES, D., SILVA, F. S. "Hot pressing effect on the shear bond strength of dental porcelain to CoCrMoSi alloy substrates with different surface treatments", Material Sciense Engineering C, v. 33, pp. 557-563, 2013.

[8] BRADEN, M., “Dental materials: 1976 literature review. Part II”, J. Dent., vol. 6, pp. 95-119, 1978.

[9] NIEVA, N., ARREGUEZ, C., CARRIZO, R. N., et al., "Bonding Strength Evaluation on Metal/Ceramic Interfaces in Dental Materials", Procedia Material Sciense., vol. 1, pp. 475-482, 2012.

[10] ORTEGA PAREDES, V. I., GÓMEZ, M. P., LÓPEZ PUMAREGA, M. I., et al., "Aplicaciones de la Emisión Acústica en la caracterización de interfaces metal/cerámico", En: Actas del II Congreso Argentino de Ingeniería - CADI 2014, Capítulo 7, T442, Tucumán, Tucumán, Argentina, 17-19 de Septiembre de 2014.

[11] GÓMEZ, M. P., Fundamentos de la Emisión Acústica, 1 ed., Buenos Aires, Argentina, 2014.

[12] NORMA MERCOSUR 302-2005, "Ensayos no destructivos .Ensayo por emisión acústica (EA) - Terminología", Primera edición (2005).

[13] NORMA ASTM E 1316-99a, "Standard Terminology for Nondestructive Examination”, American Society of Testing and Materials (1999).

[14] NORMA IRAM 779, "Ensayos no destructivos. Inspección por emisión acústica (EA). Vocabulario", Primera edición (2005). 